\title{
The recognition of anesthesiologists among the patients of surgical wards in southeastern Poland.
}

\author{
Borys M., Zyzak K., Wróblewski K., Piwowarczyk P., Czuczwar M. \\ II Department of Anesthesiology and Intensive Therapy \\ Medical University of Lublin, Poland
}

Key words: professional qualification, patient's satisfaction, respondents, anxiety, preoperative visit

\section{Background:}

The role and professional qualifications of anesthesiologists seem not to be properly recognized in society. The aim of this study was to assess patients' knowledge about anesthesiologists' qualifications.

The other objective was to evaluate the level of patients' satisfaction regarding perioperative care.

\section{Methods:}

This survey-study was performed among 200 patients of surgical wards in the southeastern Poland. The study protocol consisted eleven closeended questions concerning patients' knowledge about anesthesiologists and the assessment of preanesthetic visit and the conduit of anesthesia. Additional information obtained during the study included patients' demographic data, level of education, kind of surgery, as well as type of hospital and ward. The survey was conducted students of Medical University of Lublin, who were not involved in medical procedures.

\begin{tabular}{lll} 
Gender & Quantity & $\%$ \\
\hline Females & 115 & 57,5 \\
Males & 85 & 42,5
\end{tabular}
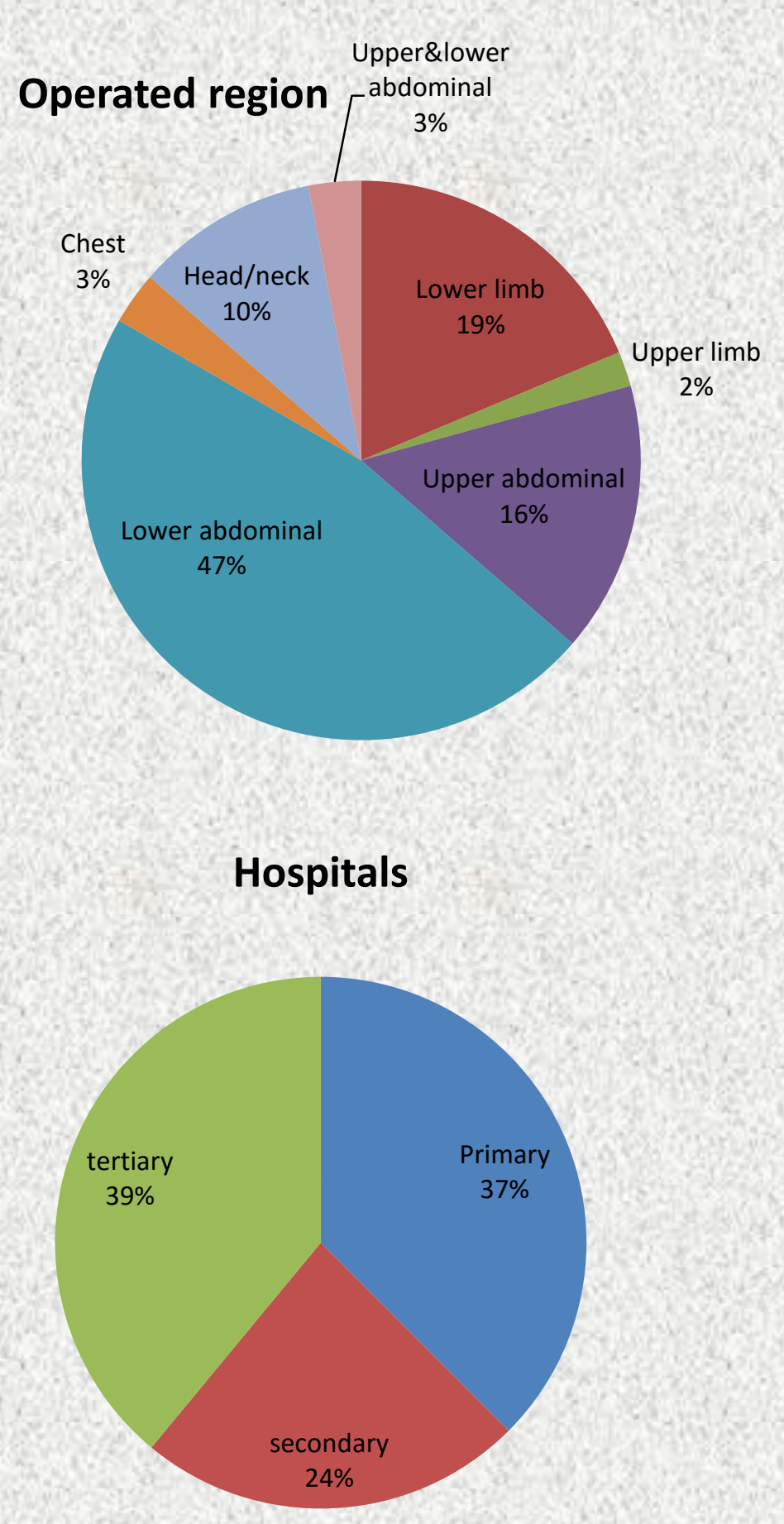

Swinhoe CF, Groves ER. Patients' knowledge of anaesthetic practice and role of anaesthetists. Anaesthesia. 1994;49:165-6.

Nagrampa D et al. A survey of anesthesiologists' role, trust in anesthesiologists, and knowledge and fears about anesthesia among predominantly Hispanic patients from an inner-city county preoperative anesthesia clinic. J Clin Anesth. 2015; 27:97-104

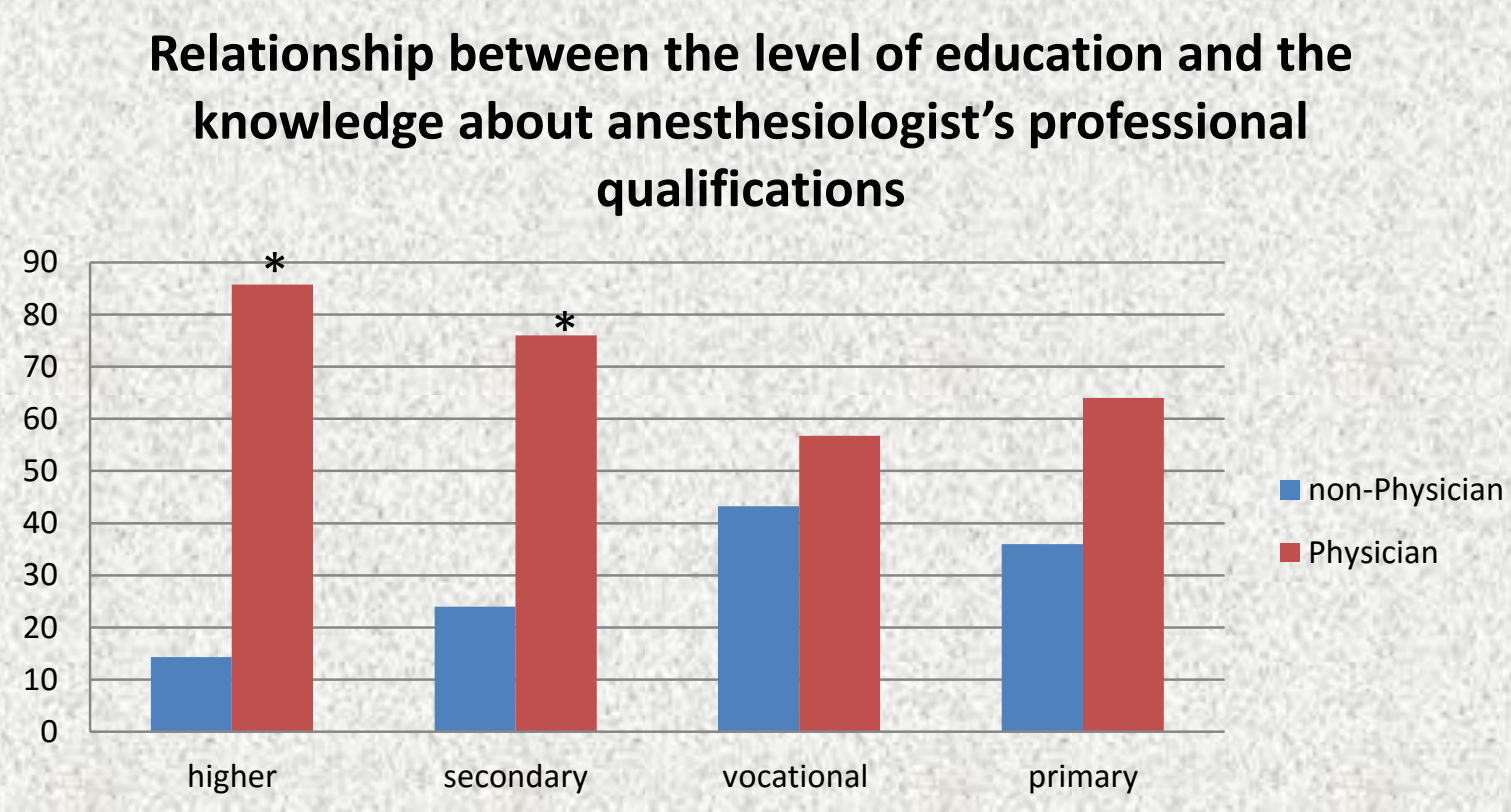

Do you remember the name of anesthesiologist?

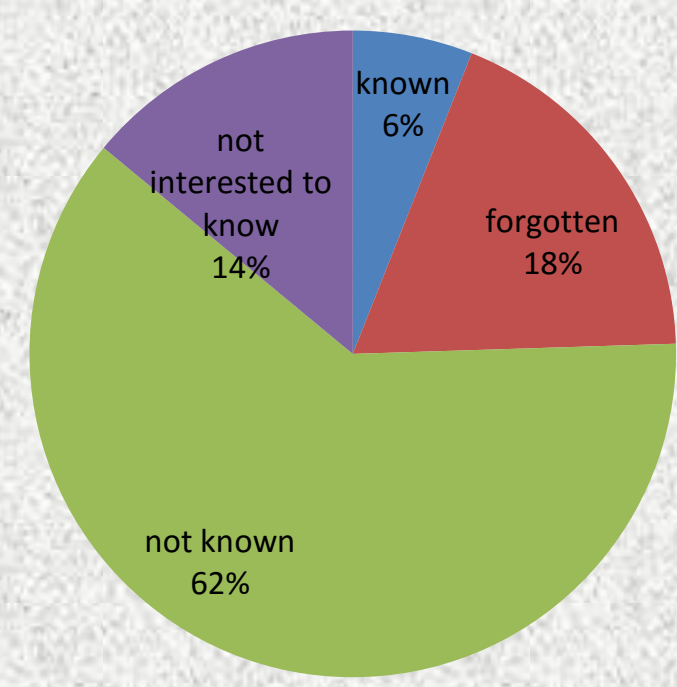

Results:

$74 \%$ of the respondents were aware of the fact that anesthesiologists are physicians. Patients with high or secondary education more often recognized an anesthetist as a doctor than people with basic one (Pearson Chi2 $=11.66649, \mathrm{p}=0.00862$ ). Patients were offered to choose the type of anesthesia in $59.5 \%$ of cases. This opportunity was more frequently given to patients in tertiary (university) hospitals (Pearson Chi2 $=17.59179, \mathrm{p}=.00734$ ). A preanesthetic visit reduced anxiety of $67,5 \%$ of patients. The quality of anesthesia was satisfactory according to $90,5 \%$ of patients.

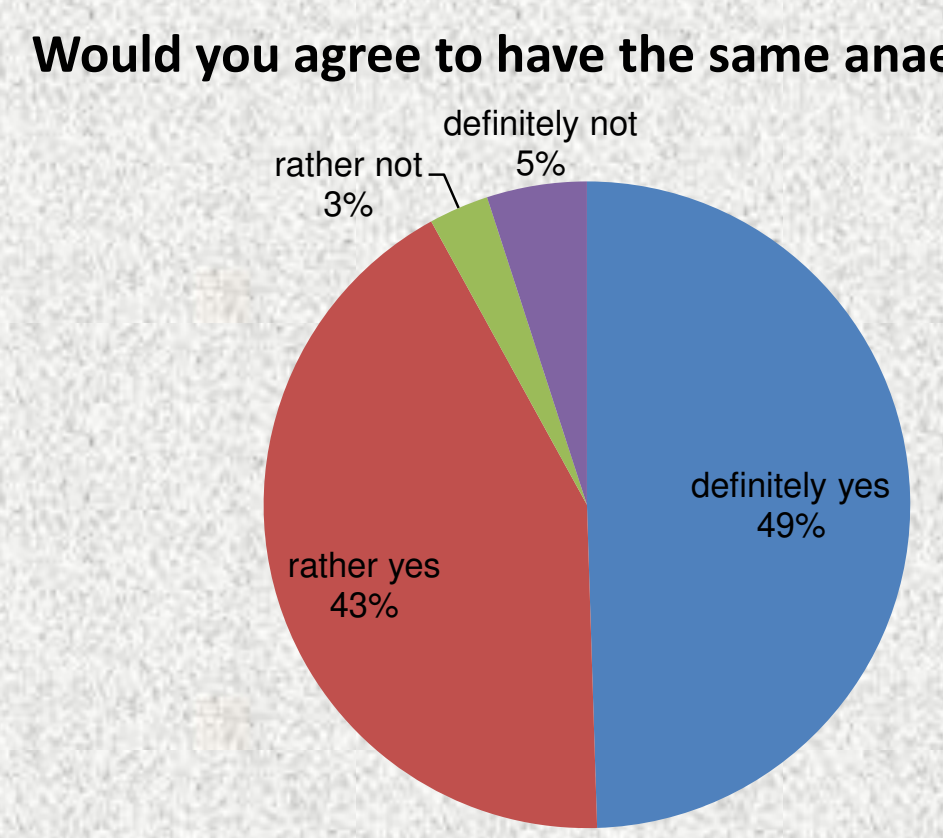

Conclusions:

In our study, the patients' knowledge about professional qualifications of anesthesiologists was similar to the data found in the literature. 